\title{
ROXAS - AN EFFICIENT AND ACCURATE TOOL TO DETECT VESSELS IN DIFFUSE-POROUS SPECIES
}

\author{
Lena Wegner ${ }^{1, *}$, Georg von Arx ${ }^{2}$, Ute Sass-Klaassen ${ }^{1}$ and Britta Eilmann ${ }^{1}$ \\ ${ }^{1}$ Forest Ecology and Forest Management Group, Centre for Ecosystem Studies, \\ Wageningen University, PO Box 47, 6700 AA Wageningen, The Netherlands \\ ${ }^{2}$ Swiss Federal Institute for Forest, Snow and Landscape Research WSL, Landscape Dynamics \\ Unit, Zürcherstr. 111, CH-8903 Birmensdorf, Switzerland \\ *Corresponding author; E-mail: lena.wegner@wur.nl
}

\begin{abstract}
Wood-anatomical parameters form a valuable archive to study past limitations on tree growth and act as a link between dendrochronology and ecophysiology. Yet, analysing these parameters is a time-consuming procedure and only few long chronologies exist. To increase measurement efficiency of wood-anatomical parameters, novel tools like the automated image-analysis system ROXAS were developed. So far, ROXAS has only been applied to measure large earlywood vessels in ring-porous species.

In this study, we evaluate if ROXAS is also suitable for efficient and accurate detection and measurement of vessels in diffuse-porous European beech. To do so, we compared the outcome of ROXAS with that of the established measurement programme Image-Pro Plus in terms of efficiency and accuracy.

The two methods differed substantially in efficiency with automatic measurements using ROXAS being 19 times faster than with Image-Pro Plus. Although the procedures led to similar patterns in annual variation of mean vessel area and vessel density, the absolute values differed. Image-Pro Plus measured systematically lower mean vessel areas and higher vessel densities than ROXAS. This was attributed to the species-specific technical settings in ROXAS, leading to more realistic results than those obtained using the default settings in Image-Pro Plus. A shortcoming of ROXAS was, however, that small vessels $\left(<100 \mu \mathrm{m}^{2}\right)$ could not be detected with sufficient accuracy. Yet, based on thin sections of European beech, it is generally difficult to distinguish such small vessels from parenchyma cells. Moreover, these small vessels do not contribute substantially to conductive efficiency. Therefore, we do not foresee any problems for most studies if the lower vessel area threshold to be measured is set to 100 $\mu \mathrm{m}^{2}$. Overall, ROXAS proved to be useful for measuring vessel parameters in diffuse-porous tree species, allowing accurate and efficient analyses of large numbers of samples.
\end{abstract}

Keywords: Wood anatomy, automatic vessel detection, novel techniques, robust chronologies. 


\section{INTRODUCTION}

Trees form a valuable archive of environmental conditions that determined tree growth, since variation in climatic conditions leave permanent imprints on the wood structure (Schweingruber 1996; Fonti et al. 2010). Especially ring width is widely used as a powerful and reliable proxy to study climate impacts on tree growth (e.g. Briffa et al. 2004). However, tree rings show an integrated response to climate conditions in the previous and the current year (Fritts 2001), making the interpretation of climate-growth relationships challenging (Kagawa et al.2006). In contrast, wood-anatomical parameters can potentially reflect climate conditions at the time of their formation which allows studying climate-growth relationships with higher time resolution (García-González \& Eckstein 2003; Fonti \& García-González 2004). Moreover, wood-anatomical parameters, such as vessel size, are strongly linked to the trees' metabolism and reveal consequences of climate limitations on tree growth. Thus, wood-anatomical parameters can act as a link between retrospective dendrochronology providing long-time series and detailed but short-term analysis of ecophysiological parameters (Fonti et al. 2010).

Despite the many potential advantages of wood-anatomical parameters, long wood-anatomical chronologies are not as widely established as tree-ring chronologies, which is mainly due to the time-consuming analysis. Thus, few long wood-anatomical chronologies exist, often focussing on big earlywood vessels only (e.g. Eilmann et al. 2009). Chen and Evans $(2005,2010)$ proposed a method for the automated measurement of vessel properties in some diffuse-porous tree species, using digital microscopy images obtained by transmitted red light. To increase measurement efficiency for woodanatomical parameters while depending on the established image analysis programme Image-Pro Plus (Media Cybernetics, Silver Spring, Maryland, USA; in the following IPP), the semi-automatic image analysis tool ROXAS was developed (von Arx \& Dietz 2005; von Arx et al. 2013). Based on species-specific settings, ROXAS automatically recognises and measures cells and annual rings, produces a suit of derived parameters, and saves all data into an MS Excel file (see von Arx 2013 for more details). ROXAS already proved to be suitable for measuring large earlywood vessels in oak with sufficient efficiency and accuracy (Fonti et al. 2009). However, it remained unclear whether ROXAS can be applied for vessel measurements in diffuse-porous tree species.

In this study, we tested if ROXAS is able to measure vessel parameters in diffuseporous European beech (Fagus sylvatica L.) with sufficient accuracy and efficiency. We measured vessel size and vessel density in nine consecutive tree rings with three different methods: (i) using ROXAS without and (ii) with manual refinement of the automatic measurement output, and (iii) using IPP with manual refinement after automatic measurement. The output of these methods was compared in terms of measurement accuracy and time efficiency.

\section{MATERIAL AND METHODS}

\section{Sampling and cross-dating of tree rings}

We analysed nine consecutive tree rings from a dominant 13-year-old European beech tree growing in a provenance trial near Wageningen, the Netherlands (latitude 
$51^{\circ} 97^{\prime} \mathrm{N}$, longitude $5^{\circ} 70^{\prime} \mathrm{E}$ ). The $5 \mathrm{~mm}$ diameter increment core was taken at breast height in summer 2011 and covered the entire diameter of the tree. Nine additional trees of the same provenance were sampled to produce a ring-width reference chronology. All ten increment cores were prepared with a core microtome (WSL Birmensdorf, Switzerland).

Tree-ring widths were measured using Lintab (Rinntech Heidelberg, Germany) and visually cross-dated to assign each ring to the corresponding calendar year (TSAP; Rinntech Heidelberg, Germany).

\section{Preparation of thin sections}

To analyse vessel size and density, we prepared thin sections with a thickness of $5 \mu \mathrm{m}$ by using a sliding microtome (WSL Birmensdorf, Switzerland). The thin sections were stained with a mixture of astrablue and safranin (150 mg astrablue, $40 \mathrm{mg}$ safranin and $2 \mathrm{ml}$ acetic acid in $100 \mathrm{ml}$ distilled water), dehydrated with a gradient of ethanol (50, 90 and 100\%) and xylol, and finally embedded permanently in Canada balsam (Table 1).

\section{Image acquisition}

Micro-images were taken (with the software Leica Application Suite v3.8) using a camera (Leica DFC320) installed on an optical microscope (Leica DM2500) and connected to a computer. To obtain images covering the whole increment core with a reasonable resolution, several overlapping photographs (taken with a $2.5 \times$ objective, image resolution: $300 \mathrm{dpi}$ ) were merged using the photo stitching software PTGui (v9.1.2) (Table 1).

Table 1. Overview of the procedures and their requirements for measuring vessels in one core (9 annual rings).

\begin{tabular}{|c|c|c|c|}
\hline Procedures & ROXAS $_{\text {auto }}$ & $\mathbf{R O X A S}_{\text {adj }}$ & IPP \\
\hline Preparation & & & \\
\hline Processes & \multicolumn{3}{|c|}{ Thin-sectioning, staining, embedding, oven treatment } \\
\hline Time & \multicolumn{3}{|c|}{$14 \mathrm{~h}$ including $12 \mathrm{~h}$ oven treatment } \\
\hline \multicolumn{4}{|l|}{ Image acquisition } \\
\hline Number of images & \multicolumn{3}{|c|}{$2-6$ per ring } \\
\hline Objective & \multicolumn{3}{|c|}{$2.5 \times$} \\
\hline Resolution & \multicolumn{3}{|c|}{300 dpi } \\
\hline Editing & \multicolumn{3}{|c|}{ Stitching with PTGui } \\
\hline Time & \multicolumn{3}{|c|}{$50 \mathrm{~min}$} \\
\hline \multicolumn{4}{|l|}{ Measurement } \\
\hline Step unit & Whole core & Whole core & Ring by ring \\
\hline Mode & Automatic & With manual correction & With manual correction \\
\hline Time $^{1}$ & $45 \mathrm{~min}$ & $9 \mathrm{~h}$ & $14 \mathrm{~h}$ \\
\hline
\end{tabular}

${ }^{1}$ Measurement times serve as an indication only. 


\section{Comparison of measurement procedures}

Vessel size and vessel density were analysed using three different procedures: (i) ROXAS automatic analysis (von Arx \& Dietz 2005; in the following ROXAS auto), (ii) ROXAS automatic analysis followed by in-built manual refinement functionality

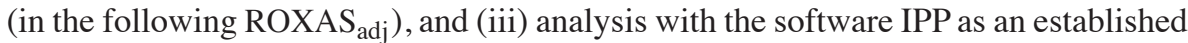
procedure (Table 1). The time required for the measurements with the three different methods was recorded.

Both ROXAS procedures initially enhanced image quality in several steps (for details see von Arx \& Dietz 2005). Vessels were then automatically detected using species-specific settings for European beech and the used staining and image-acquisition method. These settings included among others colour-, size- and shape-sensitive rules to discriminate vessels from lignified parenchyma cells and image noise. Before vessel extraction, merged candidate vessels were split. The tree-ring borders were drawn manually since an automatic separation of tree rings based on the species-specific settings was unreliable owing to the small gradient in vessel size and vessel density. After analysis, ROXAS automatically saved all data into an MS Excel file. Although the ROXAS output provided a large number of derived parameters, ranging from the number of vessels to potential hydraulic conductivity, we focussed on mean vessel area (MVA) and vessel density (VD).

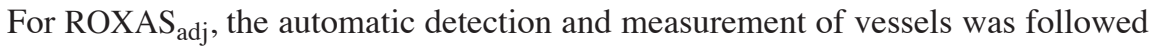
by manual correction of misidentified or unrecognised vessels using an in-built editing toolbox. Since deleting misidentified vessels was less time consuming than manually outlining unrecognised vessels, the colour and shape settings in ROXAS adj for extracting vessels were deliberately chosen to be less restrictive than in ROXAS auto $_{\text {. }}$.

For the analysis with IPP, we used the default settings. Concerning minimum and maximum vessel size, the same settings were used as for the two ROXAS procedures. Analyses were done ring by ring, using the functions "automatic [recognition of] bright objects" and "auto split". Measurement errors were corrected manually by splitting clustered vessels, deleting misrecognised vessels and drawing unrecognised vessels.

The Gleichläufigkeitskoeffizient (GLK; Eckstein \& Bauch 1969) - which reflects the percentage of oscillations in the same direction in two time series within a certain period - between MVA and VD measurements of the three tested methods was calculated to assess the accuracy of the two ROXAS measurements. Furthermore, the MVA values were used to calculate Pearson's correlation coefficient (r) between the IPP measurements and the two ROXAS measurements. For this purpose, we gradually increased the minimum vessel area to be included from 40 to $6000 \mu \mathrm{m}^{2}$ and calculated the MVA of every tree ring for the three different methods.

\section{RESULTS AND DISCUSSION}

The time required for analysis of the sample was nearly 19 times longer when using IPP in comparison to ROXAS auto $_{\text {and still } 1.5 \text { times longer than with ROXAS }}$ adj $($ Table 1). However, regarding the patterns in MVA and VD the results were quite similar for all three procedures (Fig. 1) with a GLK value of 0.75 between IPP and both ROXAS 


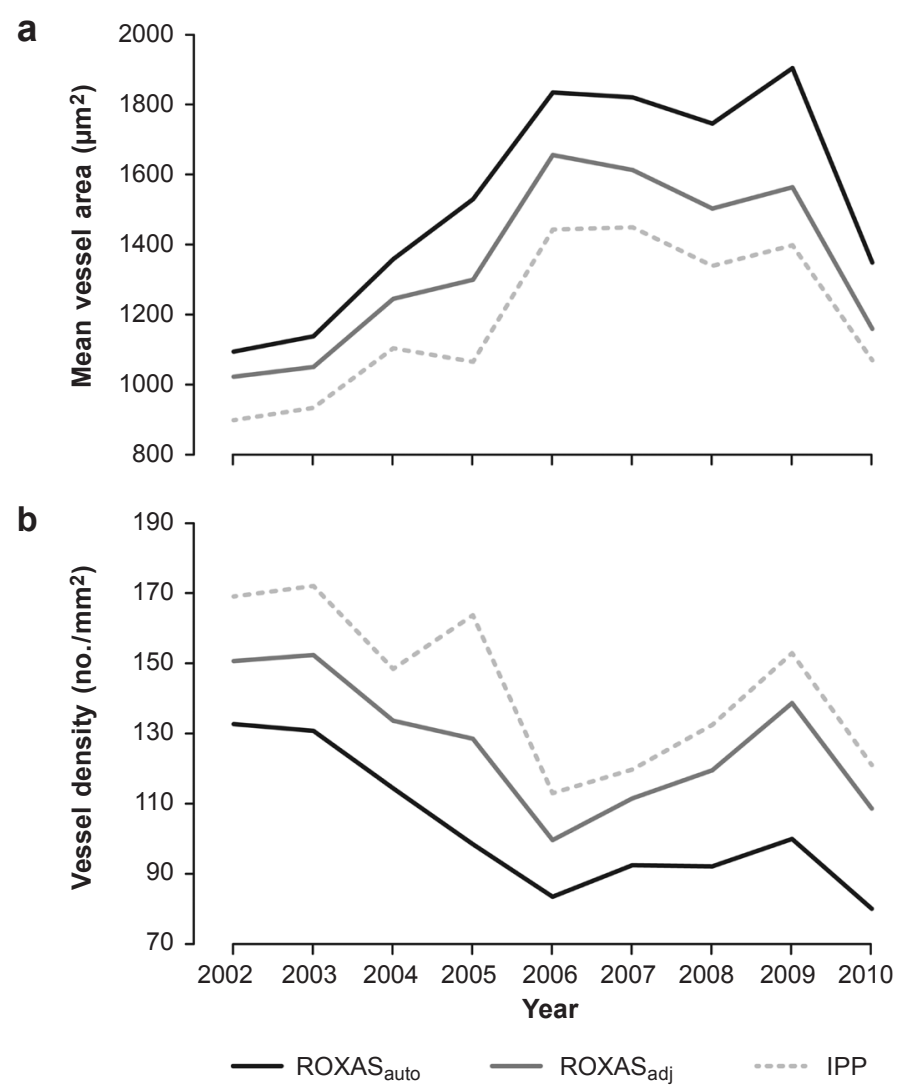

Figure 1. a: Mean vessel area and b: vessel density measured with $\operatorname{ROXAS}_{\text {auto }}, \operatorname{ROXAS}_{\text {adj }}$ and IPP.

measurements for MVA and GLK values of 0.88 and 0.63 between IPP and ROXAS adj $_{\text {anj }}$ and IPP and ROXAS ${ }_{\text {auto }}$, respectively, for VD. Still, the absolute values of MVA and VD differed systematically. IPP yielded the lowest absolute MVA values of all procedures (Fig. 1a). MVA values measured with ROXAS adj $_{\text {w }}$ were on average $13 \%$ larger than with IPP, while MVA values measured with ROXAS ${ }_{\text {auto }}$ were on average $28 \%$ larger than with IPP. The low absolute MVA values in IPP are mainly attributed to an exclusion of shadows from the cell wall in the measurements as a result of a more restrictive threshold during vessel identification. This led to a systematic underestimation of vessel size (Fig. 2a \& b). Thus, owing to the species-specific settings, the two ROXAS methods likely provide more realistic results for vessel size than the manual measurement with IPP.

The highest vessel density was detected when using IPP (Fig. 1b). VD values of ROXAS $_{\text {auto }}$ and ROXAS adj $_{\text {were on average }} 28 \%$ and $11 \%$ smaller than in IPP, owing to a better recognition of small objects in IPP. To determine the lower size threshold for an accurate detection of vessels with ROXAS, we correlated the output of IPP and 

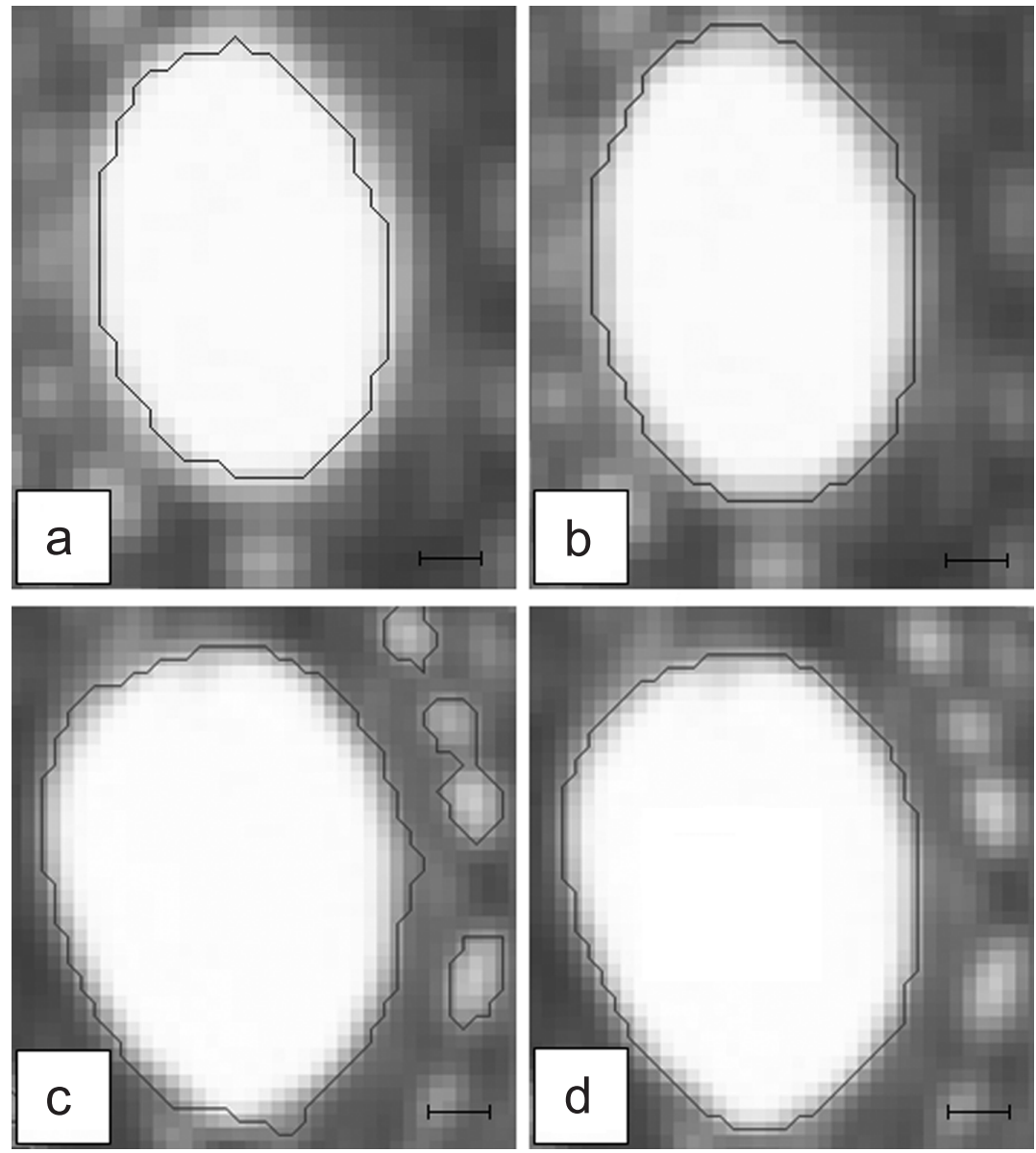

Figure 2. Different threshold values for the identification of vessels in (a) IPP and (b) ROXAS auto. Parenchyma cells identified as vessels in (c) IPP were correctly excluded with (d) ROXAS due to the shape criteria. - Scale bars $=10 \mu \mathrm{m}$.

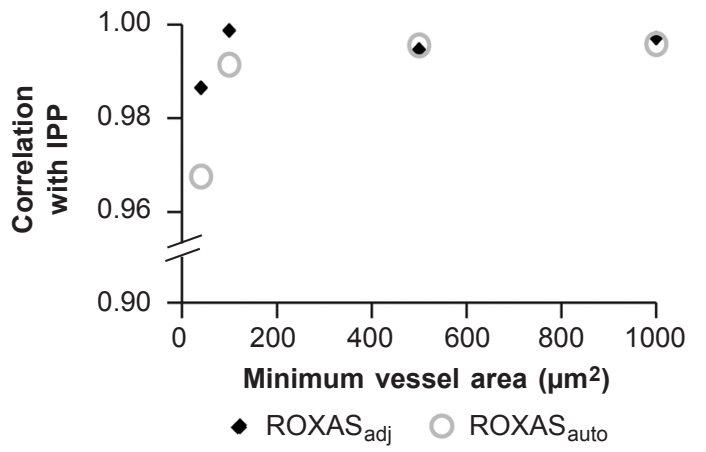

Figure 3. Correlation between mean vessel area values measured with IPP and ROXAS ${ }_{\text {auto }}$ and IPP and ROXAS $_{\text {adj }}$ depending on the minimum vessel area. 
the two ROXAS measurements and found that only very small vessels (area $<100 \mu \mathrm{m}^{2}$ ) were seemingly detected more accurately with IPP. For a minimum vessel area of $100 \mu \mathrm{m}^{2}$, correlation coefficients stabilised around 0.999 for IPP and ROXAS adj and

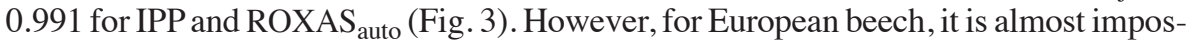
sible to distinguish vessels smaller than $100 \mu \mathrm{m}^{2}$ from parenchyma cells of similar size and shape in thin sections. Thus, the more accurate measurement with IPP below 100 $\mu \mathrm{m}^{2}$ might also be pseudo-exactness. For example, in 2005, the MVA curve based on IPP measurements showed a strong drop compared to the two ROXAS measurements (Fig. 1a). This was due to the detection of a large number of very small objects which are most likely parenchyma cells. However, owing to the similarity in cell wall structure between small vessels and parenchyma cells, the detection of these objects could not be corrected during manual editing (Fig. $2 \mathrm{c} \& \mathrm{~d}$ ). This resulted in an overestimation of VD for IPP (Fig. 1b). Owing to these discrimination problems, we advise to increase the lower vessel area measurement threshold to $100{\mu \mathrm{m}^{2}}^{2}$ in order to avoid measurement errors by parenchyma cells. Also from an ecophysiological point of view these small vessels might be negligible, since they do not contribute substantially to conductive efficiency (Sass \& Eckstein 1995; Fonti et al.2010). Moreover, based on the output of IPP in our sample, objects identified as vessels smaller than $100 \mu \mathrm{m}^{2}$ represented on average only $8.5 \%$ of the total number of vessels $(\mathrm{SD}=2.9)$ and $0.47 \%$ of the total vessel area $(\mathrm{SD}=0.19)$.

Comparing the two ROXAS measurements, ROXAS $_{\text {adj }}$ produced a more accurate

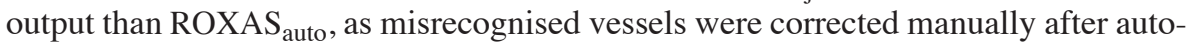
matic analysis. However, analyses with ROXAS auto $_{\text {to }}$ were substantially faster than analyses with ROXAS adj $_{\text {(Table 1). Thus, measurement errors in ROXAS }}$ auto are partly compensated for by the large number of vessels that can be efficiently measured with this method. Hence, for comparing tree rings measured with the same measurement

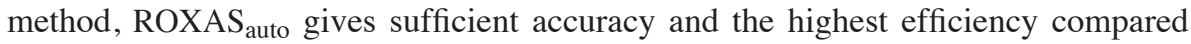
to IPP and ROXAS adj.

\section{CONCLUSION}

ROXAS is a suitable tool for the analysis of wood-anatomical parameters in diffuseporous beech, measuring vessel size as well as vessel density with sufficient accuracy. Using species-specific settings, ROXAS even provided a more realistic output than IPP with its default settings. In addition, ROXAS $_{\text {auto }}$ was much more efficient than the manual analysis methods. In summary, ROXAS can be used for the development of long and robust chronologies of wood-anatomical parameters.

\section{ACKNOWLEDGEMENTS}

We would like to thank Sven de Vries from Alterra and the EU COST project E52 'Evaluation of Beech Genetic Resources for Sustainable Forestry' for providing us with information about and access to the field trial and the beech material. We are grateful to Leo Goudzwaard for support in the field and to Ellen Wilderink for support in the laboratory. This study was conducted in the framework of the COST Action FP1106, STReESS and was funded by the Marie Curie grant for intra-European fellowships for career development within the framework of the project INPUT-drought (FP7-274085). 


\section{REFERENCES}

Briffa KR, Osborn TJ \& Schweingruber FH. 2004. Large-scale temperature inferences from tree rings: a review. Global and Planetary Change 40: 11-26.

Chen F \& Evans R. 2005. A robust approach for vessel identification and quantification in eucalypt pulpwoods. Appita J. 58: 442-447.

Chen F \& Evans R. 2010. Automated measurement of vessel properties in birch and poplar wood. Holzforschung 64: 369-374.

Eckstein D \& Bauch J. 1969. Beitrag zur Rationalisierung eines dendrochronologischen Verfahrens und zur Analyse seiner Aussagesicherheit. Forstwiss. Centralblatt 88: 230-250.

Eilmann B, Zweifel R, Buchmann N, Fonti P \& Rigling A. 2009. Drought-induced adaptation of the xylem in Scots pine and pubescent oak. Tree Physiol. 29: 1011-1020.

Fonti P, Eilmann B, García-González I \& von Arx G. 2009. Expeditious building of ringporous earlywood vessel chronologies without losing signal information. Trees 23: 665671.

Fonti P \& García-González I. 2004. Suitability of chestnut earlywood vessel chronologies for ecological studies. New Phytol. 163: 77-86.

Fonti P, von Arx G, García-González I, Eilmann B, Sass-Klaassen U, Gärtner H \& Eckstein D. 2010. Studying global change through investigation of the plastic responses of xylem anatomy in tree rings. New Phytol. 185: 42-53.

Fritts HC. 2001. Tree rings and climate. Blackburn Press, New Jersey.

García-González I \& Eckstein D. 2003. Climatic signal of earlywood vessels of oak on a maritime site. Tree Physiol. 23: 497-504.

Kagawa A, Sugimoto A \& Maximov TC. 2006. Seasonal course of translocation, storage and remobilization of $\mathrm{C}-13$ pulse-labeled photoassimilate in naturally growing Larix gmelinii saplings. New Phytol. 171: 793-804.

Sass U \& Eckstein D. 1995. The variability of vessel size in beech (Fagus sylvatica) and its ecophysiological interpretation. Trees - Structure and Function 9: 247-252.

Schweingruber FH. 1996. Tree rings and environment - dendroecology. Paul Haupt, Bern.

von Arx G. 2013. http://www.wsl.ch/roxas.

von Arx G \& Dietz H. 2005. Automated image analysis of annual rings in the roots of perennial forbs. Intern. J. Plant Sci. 166: 723-732.

von Arx G, Kueffer C \& Fonti P. 2013. Quantifying plasticity in vessel grouping - added value from the image analysis tool ROXAS. IAWA J. 34: 433-445.

Accepted: 13 August 2013 